\title{
miR-30a inhibits glioma progression and stem cell-like properties by repression of Wnt5a
}

\author{
YONGHONG ZHANG, ZHI WU, LICHAO LI and MIN XIE \\ Department of Neurosurgery, The First Hospital of Lanzhou University, Lanzhou, Gansu 730000, P.R. China
}

Received December 3, 2016; Accepted May 29, 2017

DOI: $10.3892 /$ or.2017.5728

\begin{abstract}
R-30a has been found to be dysregulated in diverse cancers and involved in the regulation of tumor progression. However, there is scarce research on the role of miR-30a in glioma. In the present study, we assessed the expression level of miR-30a in glioma tissues and cell lines. The microRNA microarray analysis revealed low expression of miR-30a in glioma tissues and cells vs. the control. Furthermore, we found that stable miR-30a inhibited cell proliferation, $G_{1}$ phase arrest and stem cell-like formation in glioma. Moreover, to investigate the molecular mechanism of miR-30a on glioma cell phenotypes, we identified Wnt5a as a new direct target gene for miR-30a by bioinformatic assay, luciferase assay and western blot analysis. Further functional studies suggested that miR-30a suppressed metastasis, sphere formation and glioma growth by targeting Wnt5a signal pathway. Collectively, our findings suggested for the first time that miR-30a may function as a tumor suppressor in glioma by targeting Wnt5a.
\end{abstract}

\section{Introduction}

With its incidence rate of almost $50 \%$, glioma is the most common form among CNS gliomas. Although new therapeutic approaches have been developed, there are still many common problems that need to be resolved including therapy resistance to improve long-term survival. The origin of gliomas is largely unknown but there is increasing speculation that they might arise from glioma stem cells (GSCs), which might consist of transformed neural stem cells (1-4). Recent advances in our understanding of the biological features of glioma offer opportunities to the design of a new therapeutic strategy based on targeting essential signaling pathways.

Correspondence to: Dr Yonghong Zhang, Department of Neurosurgery, The First Hospital of Lanzhou University, Donggang West Road 1, Lanzhou, Gansu 730000, P.R. China

E-mail: yonghong_zhang@cntv.cn

Key words: glioma, metastasis, Wnt5a, miR-30a, stem cell, proliferation
MicroRNAs (miRNAs) are a family of endogenous small non-coding RNAs that regulate gene expression via the sequence-specific base pairing on the 3'-untranslated regions (3'UTRs) of target mRNAs, leading to mRNA cleavage or translation inhibition (5). A great number of the human miRNAs function either as oncogenes or as tumor suppressors. Thus, their function acting as tumor-suppressor or carcinogenic miRNAs may vary depending on their targets (6-8), resulting in influencing glioma formation and growth. The miRNAs have been reported to be aberrantly overexpressed or downregulated during glioma progression, including miR-20a and miR-106a (9), miR-29a (10), miR-145 (11), miR-656 (12), miR-300 (13), miR-16 (14), miR-34a (15), miR-503 (16), miR-203 (17) miR-100 (18), miR-26a (19), miR-23b (20) and miR-218 (21). These miRNAs play oncogenic or tumorsuppressive roles in the regulation of cell growth, migration and invasion by repressing their target genes.

miRNA 30a (miR-30a) is a member of the miR-30 family, which consists of six distinct mature miRNA sequences. There is considerable evidence suggesting that the dysregulation of miR-30a is correlated with several types of malignant tumors, including breast, lung, thyroid, gastric cancer and leukemia (22). Metadherin (23), SNAIL1 (24), Beclin-1 (25) and PIK3CD (26) are potential targeted genes of miR-30a, which promotes glioma progression. However, there are relatively few studies available that report a role for miR-30a in tumorigenesis and progression of glioma. In the present study, we investigated the potential involvement of miR-30a by examining its expression and its effects on tumorigenesis, cell growth, cell cycle distribution, colony formation, migration, invasion and stem cell-like properties in glioma. Thus, mechanistic investigation revealed that miR-30a was shown to control Wnt5a expression in progression of glioma as a tumor suppressor.

\section{Materials and methods}

Clinical samples. Primary glioma tissue samples and normal samples were obtained from the patients in the First Hospital of Lanzhou University (Lanzhou, China). None of the patients had received either radiotherapy or chemotherapy. Both tumor and normal tissues were histologically confirmed by $\mathrm{H} \& \mathrm{E}$ (hematoxylin and eosin) staining. The tumor tissues derived from 16 cases with glioma, and the normal tissues derived from patients with brain injury. Informed consent was obtained 
from each patient and the research protocols were approved by the Ethics Committee of Lanzhou University Hospital.

Cell culture. Human glioma cells T98G, SHG44, U251, U87 and U373 were obtained from the American Type Culture Collection (ATCC; Manassas, VA, USA) were cultured in Dulbecco's modified Eagle's medium (DMEM) supplemented with $10 \%$ fetal bovine serum (FBS), $100 \mathrm{U} / \mathrm{ml}$ penicillin and $100 \mu \mathrm{g} / \mathrm{ml}$ streptomycin. All cells were cultured at $37^{\circ} \mathrm{C}$ in a humidified atmosphere containing $5 \% \mathrm{CO}_{2}$. Human normal cells HA (ScienCell Research Laboratories, Carlsbad, CA, USA) isolated from human brain (cerebral cortex) were cultured in microglia medium with $10 \%$ FBS.

Oligonucleotide synthesis and lentiviral transduction. The oligonucleotide of mature miR-30a antagomir was chemosynthesized, amplified and cloned into GV232-Puro Vectors by Shanghai Genechem, Co., Ltd. (Shanghai, China). The correct sequences and insertions were confirmed by DNA sequencing. Cells were lentivirus-transfected with either the GV232-Puro-miR-30a recombined vector (LV-miR-30a) or emptyGV232-Puro vector (negative control, miR-control) using Lipofectamine 2000 transfection reagent (Invitrogen, Carlsbad, CA, USA). The transduced cells with a cell density of over $40 \%$ confluency were exposed to puromycin dihydrochloride (1 mg/ml; Sigma-Aldrich, St. Louis, MO, USA) for resistance selection. Stable cell lines were selected with $0.5 \mathrm{mg} / \mathrm{ml}$ puromycin in the first round of selection. Lentivirus-mediated silencing of miR-30a was verified by quantitative reverse transcription-PCR (qRT-PCR) and western blot analysis.

miRNA target validation. A fragment of Wnt5a 3'UTR (untranslated regions) was amplified by PCR and cloned downstream of the firefly luciferase gene in pGL4 vector (Promega, Madison, WI, USA). The vector was named wild-type 3'UTR. Site-directed mutagenesis of the miR-30a binding site in Wnt5a 3'UTR was performed using GeneTailor Site-Directed Mutagenesis system (Invitrogen) and named mutant 3'UTR.

RNA isolation and real-time $R T-P C R$. Total RNA was extracted using TRIzol reagent (Invitrogen) following the instructions. Briefly, the cells were lysed in TRIzol and then mixed with chloroform. The lysate was centrifuged to separate RNA, DNA and protein, the total RNA recovered was precipitated with isopropanol, washed in $75 \%$ ethanol to remove impurities before dissolved in water. Subsequently, $2 \mu \mathrm{g}$ of RNA was treated with DNase to remove contaminating DNA prior to reverse transcription to cDNA using $S Y B R^{\circledR}$ PCR kit (Takara Bio, Shiga, Japan). With the primers purchased from Invitrogen, real-time RT-PCR was performed using a sequence detector (ABI Prism; Applied Biosystems, Foster City, CA, USA) to measure mRNA expression. The relative expression levels were quantified by comparing $\mathrm{Ct}$ values of the samples with those of the reference, the data were normalized to the internal control GAPDH.

Cell viability assay. To detect the growth of glioma cells and the growth curve, cell viability was assessed by MTT assay. Logarithmic phase cells were seeded in 96-well culture plates at a density of $5 \times 10^{3}$ cells/well [the edge wells of the plate are filled with aseptic phosphate-buffered saline buffer (PBS)]. The cells were incubated at $37^{\circ} \mathrm{C}, 5 \% \mathrm{CO}_{2}$ until cells covered the bottom of the well. A total of $20 \mu \mathrm{l}$ of the MTT solution was added to each well (5 mg/ml, $0.5 \%$ MTT) and the cells were cultured for $4 \mathrm{~h}$ at $37^{\circ} \mathrm{C}$. After the incubation, the supernatant was discarded and $150 \mu \mathrm{l}$ dimethyl sulfoxide was added to each well. Afterwards, the culture plate was shaken at low speed for $10 \mathrm{~min}$ until the crystals dissolved completely. The ELISA reader was used to measure the absorbance at $570 \mathrm{~nm}$.

Colony formation assay. Cells in logarithmic growth phase were digested in $0.5 \%$ trypsin/ $0.04 \%$ EDTA and single cell suspension was prepared. Then, these cells were added to 6 -well plates (200 cells/well) followed by incubation at $37^{\circ} \mathrm{C}$ in a humidified incubator containing $5 \% \mathrm{CO}_{2}$ for $24 \mathrm{~h}$. Non-adherent cells were removed. After culture for 10-14 days, colonies were present. These cells were seeded into 96-well plates followed by incubation at $37^{\circ} \mathrm{C}$ in an environment with saturated humidity and $5 \% \mathrm{CO}_{2}$. The colony formation efficiency and the morphology of colonies were photographed using a microscope. The colony size and cells in each colony were measured.

Cell cycle assay. Total cells were collected by trypsinization. Bovine pancreatic RNAse (Wuhan Boster Biological Technology, Ltd., Wuhan, China) was added at a final concentration of $2 \mu \mathrm{g} / \mathrm{ml}$, incubated at $37^{\circ} \mathrm{C}$ for $30 \mathrm{~min}$, then $20 \mu \mathrm{g} / \mathrm{ml}$ propidium iodide (PI) was added and incubated for $20 \mathrm{~min}$ at $25^{\circ} \mathrm{C}$. Cells $\left(5 \times 10^{4}\right)$ were analyzed by FACSCalibur flow cytometer (Becton-Dickinson, Franklin Lakes, NJ, USA) in each group.

Western blot analysis. Total cells were lysed using the RIPA (Bio-Rad Laboratories, Inc., Philadelphia, PA, USA) buffer (50 mM Tris- $\mathrm{HCl}, \mathrm{pH} 8.0,150 \mathrm{mM} \mathrm{NaCl}, 1 \mathrm{mM}$ dithiothreitol, $0.1 \%$ SDS). Proteins were separated by SDS-PAGE and transferred to polyvinylidene difluoride (PVDF) membranes (Millipore, Bedford, MA, USA) at $55 \mathrm{~V}$ for $4 \mathrm{~h}$ at $4^{\circ} \mathrm{C}$. After blocking, the membranes were incubated with primary antibodies overnight at $4^{\circ} \mathrm{C}$, washed three times with TBS Tween-20, and followed by secondary antibodies conjugated with horseradish peroxidase at 1:5,000 dilution in TBS for $1 \mathrm{~h}$ at $25^{\circ} \mathrm{C}$. Western blotting were visualized on X-ray film by an automated chemiluminescence system.

Luciferase assay. For reporter assays, wild-type or mutation 3'UTR vector and the control vector pRL-CMV (cytomegalovirus; coding for Renilla luciferase; Promega) were cotransfected. Luciferase activity was measured $36 \mathrm{~h}$ after transfection. Firefly and Renilla luciferase reporter activity was measured using Luc-Pair Duo-luciferase Assay kit 2.0 as per manufacturer's instructions.

Migration assay. In order to evaluate the effect of metastatic properties, Transwell migration assays was conducted. Glioma cells $\left(1 \times 10^{5}\right)$ were placed in the top chamber onto the noncoated membrane and allowed to migrate toward DMEM medium containing fetal calf serum (FCS) in the lower chamber. After incubation with methanol, cells were fixed for 

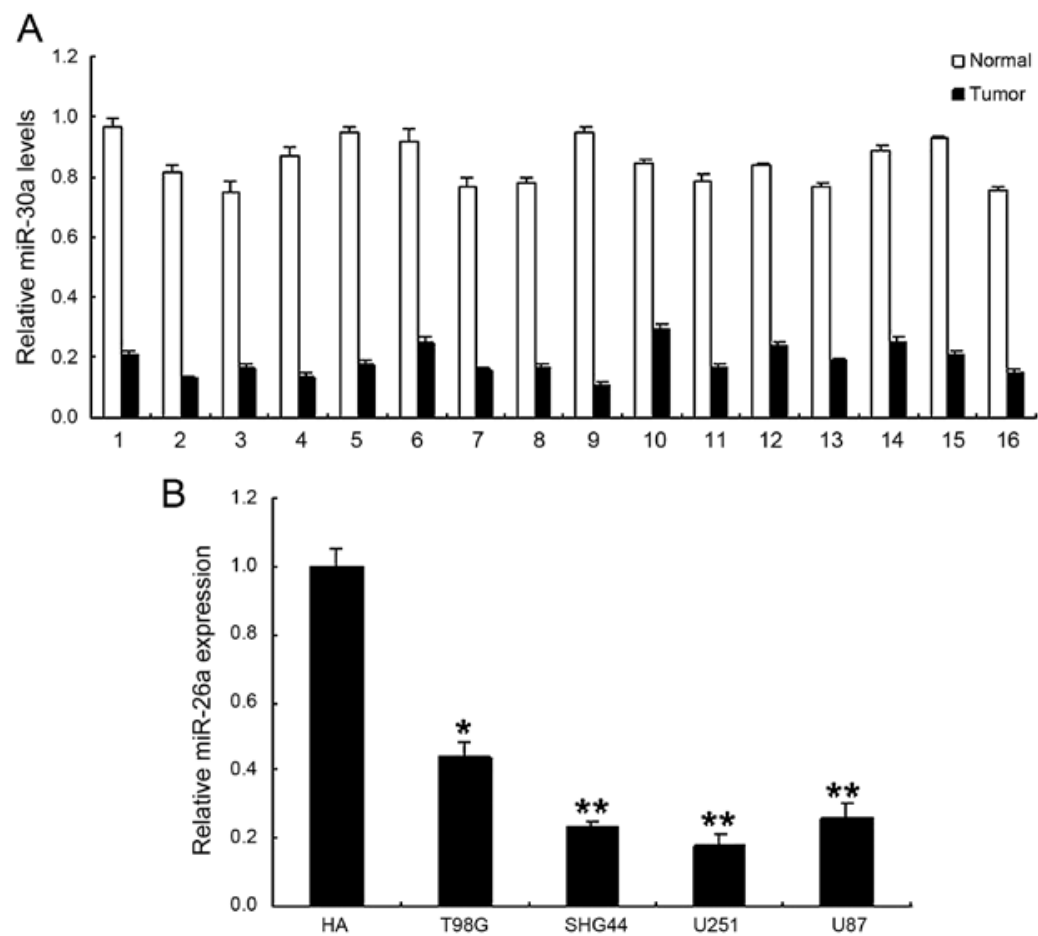

Figure 1. Decreased expression of miR-30a in human glioma. (A) The expression level of miR-30a in human glioma samples ( $\mathrm{n}=16)$ and control tissues ( $\mathrm{n}=16$ ). (B) RT-PCR analysis of miR-30a expression in T98G, SHG44, U251, U87 and U373 glioma cells and HA cells. ${ }^{* *} \mathrm{P}<0.01,{ }^{*} \mathrm{P}<0.05$.

$24 \mathrm{~h}$ and stained with $0.1 \%$ crystal violet (Sigma-Aldrich). The number of cells was counted using a light microscope.

Invasion assay. In order to evaluate the effect of metastatic properties, invasion assay was conducted. Cells $\left(1 \times 10^{5}\right)$ were placed in the top chamber onto the Matrigel coated membrane. Each well was coated freshly with Matrigel $(60 \mu \mathrm{g}$; BD Biosciences, San Jose, CA, USA). Cells were placed in serumfree medium or growth factors, and medium containing serum was used as a chemoattractant in the lower chamber. After incubating for $48 \mathrm{~h}$, cells that did invade via the pores were removed by a cotton swab. Cancer stem cells (CSCs) on the lower surface of the membrane were fixed in methanol and then stained with crystal violet. The number of cells was counted using a light microscope (27).

Tumor sphere assay. Sphere formation assay (27) was performed as described. In brief, cells were plated in 6-well ultralow attachment plates (Corning Inc., Corning, NY, USA) at a density of $1 \times 10^{3}$ cells $/ \mathrm{ml}$ in DMEM supplemented with 1\% N2 supplement (Invitrogen), 2\% B27 supplement (Invitrogen), $20 \mathrm{ng} / \mathrm{ml}$ human platelet growth factor (SigmaAldrich), $100 \mathrm{ng} / \mathrm{ml}$ epidermal growth factor (Invitrogen) and $1 \%$ antibiotic-antimycotic (Invitrogen) at $37^{\circ} \mathrm{C}$ in a humidified atmosphere containing $5 \% \mathrm{CO}_{2}$. Sphere were collected after 7 days and dissociated with accutase (Innovative Cell Technologies, Inc., San Diego, CA, USA). The cells obtained from dissociation were sieved through a $40-\mu \mathrm{m}$ filter, and counted by coulter counter using trypan blue dye.

Mouse xenograft models. The mice (6-weeks-old) were purchased from Beijing Weitongli, Co., Ltd. (Beijing, China). A total of $1.0 \times 10^{6}$ cells, either stably expressing glioma cells
LV-miR-30a or LV-miR control and LV-miR-30a with Wnt5a overexpression or LV-miR control with Wnt5a overexpression, were injected subcutaneously into the abdomen of each mouse, respectively. After the tumors were $\sim 100 \mathrm{~mm}^{3}$, mice were examined for the effects of tumor burden and tumor growth, every two days and tumor measurements were performed weekly. Tumor volume was calculated using the formula: Tumor volume $=\left[\right.$ length $\mathrm{x}$ width $\left.{ }^{2}\right] / 2$ as previously reported . Approximately 3 weeks after inoculation, the mice were euthanized by subcutaneous injection with sodium pentobarbital $(40 \mathrm{mg} / \mathrm{kg})$ and the tumors were weighed, all mice were handled according to the protocol approved by the Committee on the Ethics of Animal Experiments of the hospital. All tumors were dissected, and sizes and weights were measured and recorded (28).

Statistical analysis. All data were expressed as the mean \pm SD of at least three independent experiments. Differences between the groups were analyzed by one- or two-way ANOVA, followed by Bonferoni's multiple comparison tests using PRISM statistical analysis software (GrafPad Software, Inc., San Diego, CA, USA). Differences at $\mathrm{P}<0.05$ were considered as significance level.

\section{Results}

Low expression of miR-30a in human glioma. To explore the possible action of miR-30a in glioma, the expression of miR-30a was examined and quantified by real-time RT-PCR at both tissue and cell levels. We examined the expression of miR-30a in 16 cases with glioma and their compared normal tissues. As shown in Fig. 1A, the expression levels of miR-30a in glioma samples were lower than those in normal samples. 
A

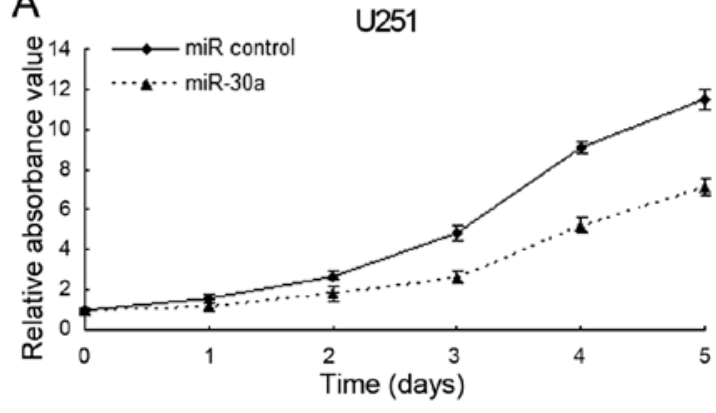

C
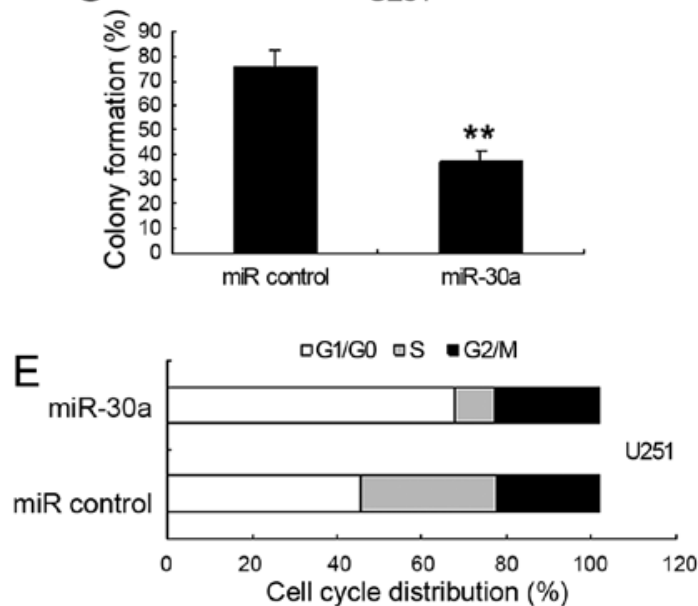

B

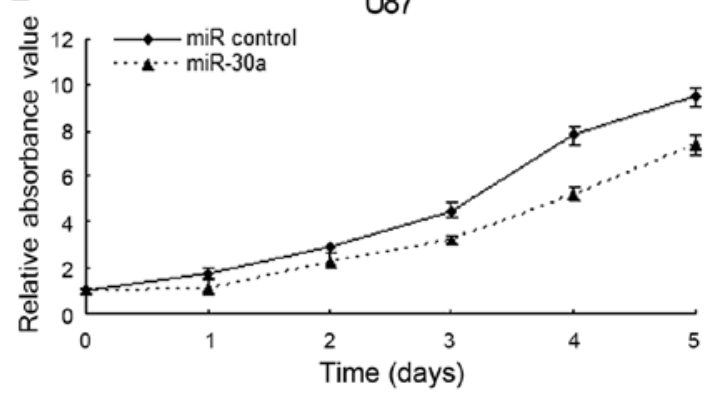

U87
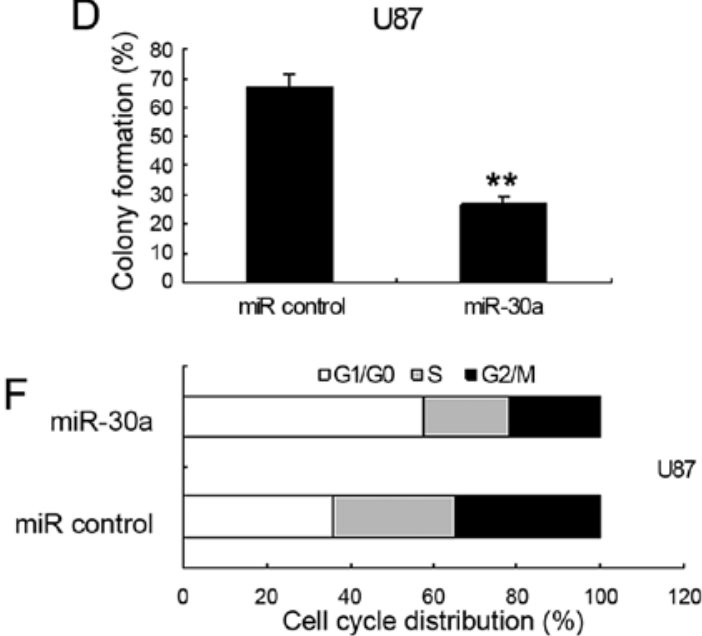

Figure 2. Enforced expression of miR-30a induced growth inhibition in glioma in vitro. (A) Effect of miR-30a on cell proliferation was measured by MTT assay after miRNA infection in U251 cells. (B) Effect of miR-30a on cell proliferation was measured by MTT assay after miRNA infection in U87 cells. (C) Effect of miR-30a on cell proliferation was measured by colony formation assay after miRNA infection in U251 cells. (D) Effect of miR-30a on cell proliferation was measured by colony formation assay after miRNA infection in U87 cells. (E) Cell cycle distribution of U251 cells infected with miRNAs for $48 \mathrm{~h}$. (F) Cell-cycle distribution of U87 cells infected with miRNAs for $48 \mathrm{~h} .{ }^{* *} \mathrm{P}<0.01,{ }^{*} \mathrm{P}<0.05$.
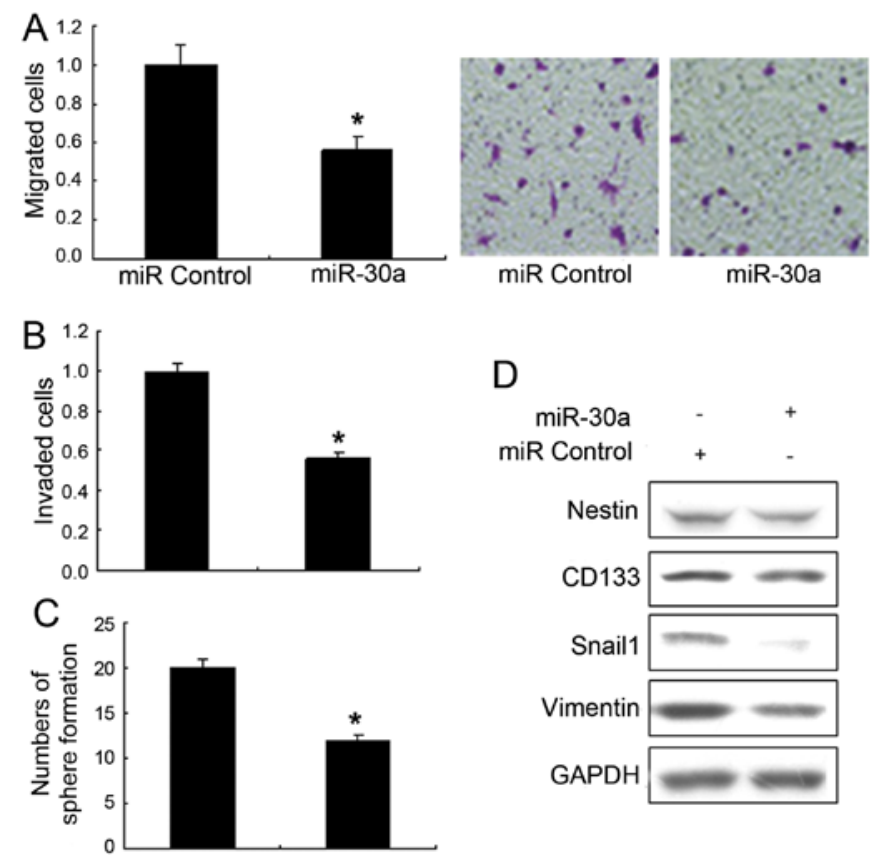

Figure 3. miR-30a suppresses glioma metastasis and stem cell-like properties. (A) Migration of U251 cells with miR-30a and miR-control. (B) Invasion of U251 cells with miR-30a and miR-control. (C) Tumor sphere formation of U251 cells with miR-30a and miR-control by sphere formation assay. ${ }^{* *} \mathrm{P}<0.01,{ }^{*} \mathrm{P}<0.05$. (D) miR-30a suppressed stem cell markers in glioma cells. Markers such as CD133, nestin and Snail1 were assayed by western blotting.
Similarly, miR-30a in human glioma cells was shown significantly reduced comparing with control cells (Fig. 1B). It is suggested that miR-30a may function as a tumor suppressor in the progression of human glioma.

miR-30a suppresses proliferation and mediates the accumulation of $G_{1}$-phase glioma cells. To investigate the effect of miR-30a on cell proliferation, U87 and U251 cells were transfected with LV-miR-control or LV-miR-30a, respectively. MTT and colony formation assay showed that miR-30a inhibited cell proliferation in U251 cells (Fig. 2A and C) and U87 cells (Fig. 2B and D). In addition, there was no difference on cell proliferation between cells with the LV-miR-control and the control cells (data not shown). To further ascertain miR-30a mediating growth inhibition, cells with LV-miR-30a and cell cycle distribution were examined. U251 cells infected with LV-miR-30a had an increased percentage of cells in $\mathrm{G}_{1}$ phase but fewer cells in $S$ phase comparing with the control (Fig. 2E and F). As shown in Fig. 2, there was a correlation between the growth-suppressive effect of miR-30a and the $\mathrm{G}_{0} / \mathrm{G}_{1}$ phase arrest. Therefore, the accumulation of $\mathrm{G}_{1}$-phase glioma cells mediated by miR-30a is as a direct cause of the cell proliferation inhibition.

miR-30a inhibits stem cell-like properties in glioma. To investigate the effects of miR-30a on glioma metastasis and stem 


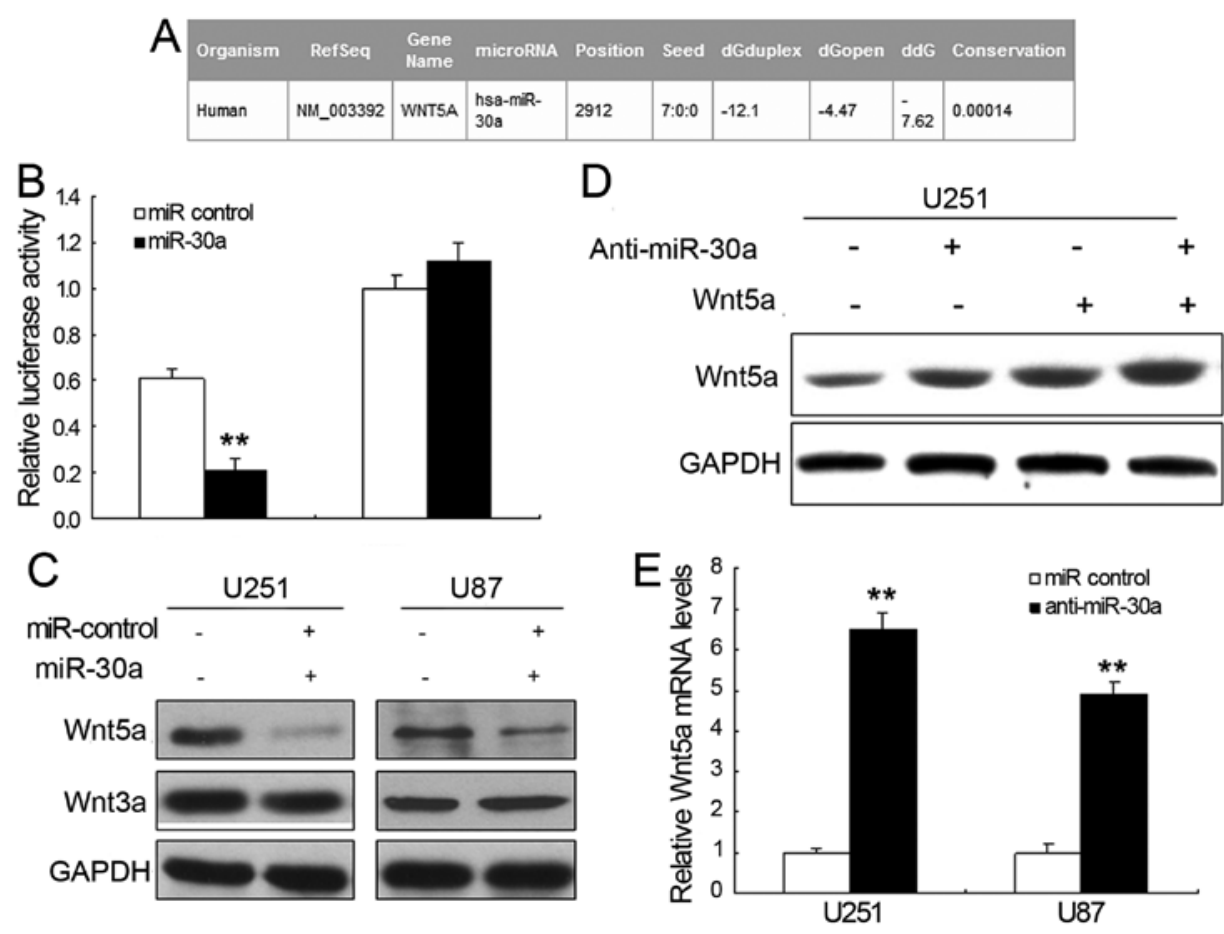

Figure 4. Restoration of miR-30a downregulates Wnt5a expression. (A) The 3'-UTR of the Wnt5a gene contains binding sites for miR-30a according to bioinformatic analysis. (B) miR-30a suppressed the expression of a luciferase reporter gene harbouring the 3'UTR of Wnt5a comparing with Wnt5a 3'UTR mutation. The data presented are shown as means \pm SD collected from three independent experiments. (C) miR-30a restoration downregulated Wnt5a in glioma cells at the protein level. (D) The expression of Wnt5a increased in the cells with anti-miR-30a. (E) miR-30a restoration downregulated Wnt5a in glioma cells at mRNA level. ${ }^{* * *} \mathrm{P}<0.01,{ }^{*} \mathrm{P}<0.05$.

cell-like properties, the Transwell migration assays and invasion assays of U251 cells with LV-miR-30a were performed. It is shown that upregulation of miR-30a significantly decreased migration (Fig. 3A) and invasion (Fig. 3B) exposed to TGF- $\beta$. The sphere formation of U251 cells with LV-miR-30a was much lower than the control (Fig. 3C). Metastasis associated markers were also detected by western blot analysis and the results showed that vimentin and SNAIL1 decreased in the U251 cells with LV-miR-30a (Fig. 3D). The above indicated that miR-30a prevented the glioma cell from stem-like cells.

Wnt5a is a target gene for miR-30a. To further explore the possible molecular mechanisms of miR-30a-mediated glioma progress inhibition, we applied the bioinformatic analysis to search the potential targets of miR-30a. It is shown that Wnt5a is considered to be directly suppressed by miR-30a (Fig. 4A). The luciferase activity of pGL4-Wnt5 $\alpha$-WT in U251 cells was much lower than in control cells (Fig. 4B). Moreover, pGL4-Wnt5a-Mut luciferase activity was rescued. Furthermore, we examined whether miR-30a could regulate the expression of endogenous Wnt5a in U251 cells. Comparing with the control, the mRNA levels of endogenous Wn5a (Fig. 4C) were downregulated when cells were transected with miR-30a. The expression of Wnt5a increased in the cells with anti-miR-30a (Fig. 4D and E). These data indicated that Wnt5a acted as a new target gene for miR-30a.

miR-30a inhibits metastasis and sphere formation by targeting Wnt5a signal pathway in glioma cells. In view of the fact that Wnt5a was the latent target of miR-30a, overexpression of Wnt5a was performed to test whether miR-30a regulates metastasis and sphere formation by targeting Wnt5a signal pathway in glioma cells. It is suggested that miR-30a in U251 cells inhibited cell migration and invasion with Wnt5a overexpression (Fig. 5A and B). Sphere formation assay also demonstrated that miR-30a inhibited the self-renewal ability of glioma cells (Fig. 5C). Since Wnt signaling pathway is an important pathway involved in primary glioma, we then detected several downstream proteins in Wnt signaling pathway. The protooncogene cMyc, downstream of the Wnt singling pathway, was decreased by miR-30a overexpression. A similar result was obtained for phosphorylated JNK, an important downstream protein of Wnt5a (Fig. 5D).

miR-30a inhibits glioma growth by targeting Wnt5a signal pathway in vivo. Based on the above effect of miR-30a observed in in vitro experiments, we further investigate miR-30a mediating growth inhibition in vivo. The xenograft model of glioma in nude mice was applied using U251-miR-30a and U251-miR control. Next, we measured the tumor size every 5 days and plotted the growth curve against the average tumor size. The results showed that miR-30a suppressed glioma growth in vivo (Fig. 6A).

\section{Discussion}

Although previous studies have shown that miR-30a may act as a tumor suppressor or oncogene, the detailed mechanism of miR-30a involvement in glioma is not well understood. In the present study, we found that the glioma tissues had a significant downregulation of the expression of miR-30a comparing with the normal tissues. From this finding, we 

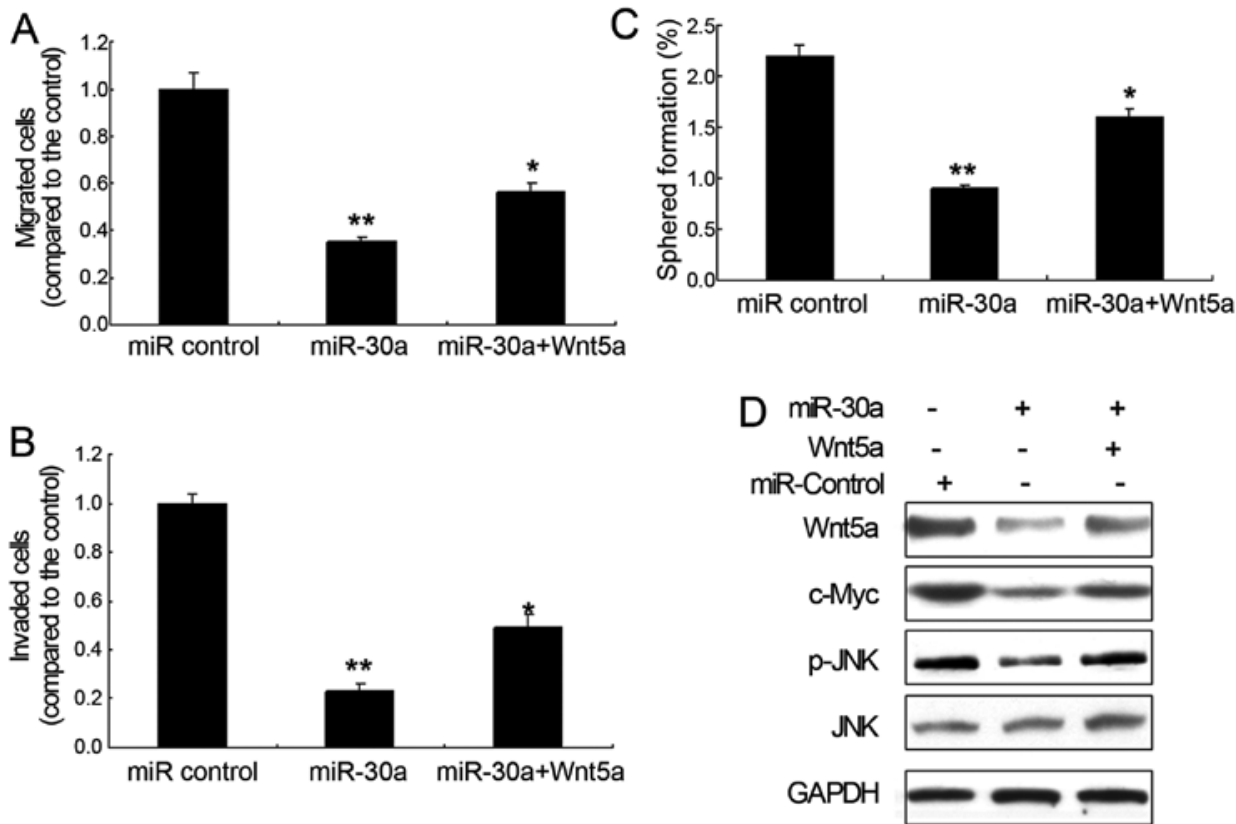

Figure 5. miR-30a suppresses glioma cell progression targeting Wnt5a. (A) miR-30a suppressed migration of Wnt5a overexpression glioma cells. (B) miR-30a suppressed invasion of Wnt5a overexpression glioma cells. (C) miR-30a inhibited tumor sphere formation of glioma cells with Wnt5a overexpression. (D) miR-30a inhibited Wnt5a signal pathway in glioma cells. ${ }^{* *} \mathrm{P}<0.01,{ }^{*} \mathrm{P}<0.05$.
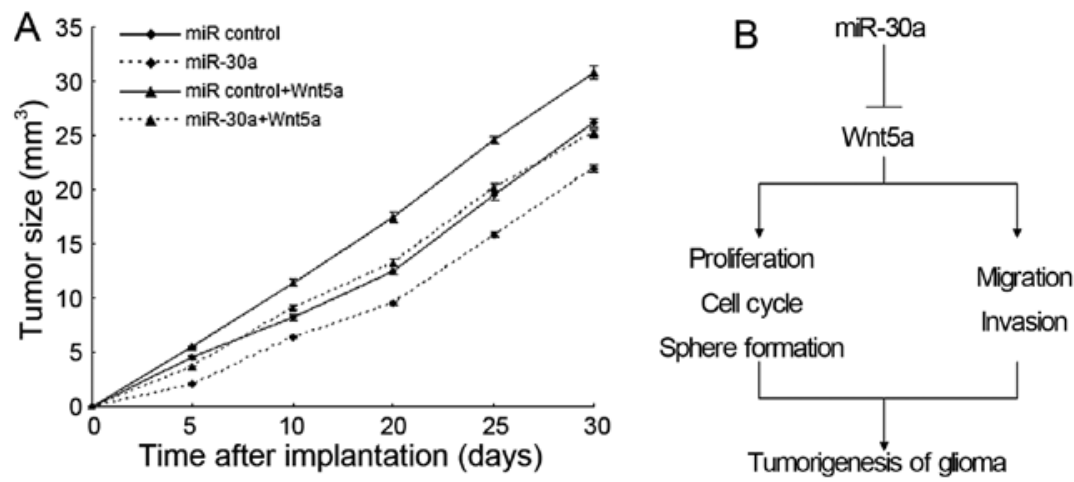

Figure 6. miR-30a inhibits glioma by targeting Wnt5a signal pathway in vivo. (A) Tumor growth on nude mice set up using glioma LV-miR-30a cells or LV-miR control and LV-miR-30a with Wnt5a overexpression or LV-miR control with Wnt5a overexpression. (B) A diagram of miR-30a signaling pathways for Wnt5a control in glioma.

aimed to clear the suppressor role of miR-30a in glioma Thus, we also discovered that miR-30a induced $\mathrm{G}_{1}$ arrest of glioma cells and suppressed cell proliferation. Our finding suggested that miR-30a suppressed the progression of glioma by targeting Wnt5a for the first time. In addition, overexpression of miR-30a in U251 cells restrained oncogenesis in nude mice, which showed that miR-30a acts as a tumor suppressor in glioma.

Over the past few decades, many studies have focused on the cancer-related role of miR-30 family. This family is widely known to be implicated in various cellular processes including cell differentiation, organ development and substance metabolism $(29,30)$. Several studies indicated that miR-30a could regulate breast cancer cell proliferation and migration and reduce the oncogenic abilities of breast and lung cancer depending on their targets (31-33). In order to investigate potential target genes of miR-30a, we used bioinformatics analyses to search the latent target genes and than discovered that Wnt5a was a novel potential target gene of miR-30a. Wnt family members are classified into two groups: Wnt1, Wnt3a and Wnt7a, activate the $\beta$-catenin pathway, and have been shown to be present in mammals $(34,35)$. Another group including Wnt2, Wnt4, Wnt5a, Wnt5b, Wnt6 and Wnt11, activate a $\beta$-catenin-independent pathway that primarily regulates cell motility and polarity $(34,35)$. Moreover, $\beta$-catenin-independent pathway is known to activate various protein kinases including protein kinase $\mathrm{C}(\mathrm{PKC}), \mathrm{Ca}^{2+}$ calmodulin-dependent protein kinase II, Rho-associated kinase and c-jun N-terminal kinase (JNK). In particular, Wnt5a is a representative of the Wnt family that activate the $\beta$-catenin-independent pathway and distinct routes $(36,37)$. Previous research showed that Wnt5a stimulates proliferation and migration in cancer cells. Moreover, Wnt5a expression is related with the aggressiveness of ocular melanoma, lung, breast and gastric cancer, indicating that Wnt5a has oncogenic properties $(36,37)$. A recent study showed that the glioma cells 
were found to express significantly high levels of Wnt5a. It was also suggested that Wnt5a promotes invasion activities of glial cells at least via the activation and expression of JNK and matrix metalloproteinase-1 (MMP-1) (37). In this study, we proved that Wnt5a was a novel target gene of miR-30a that could inhibit metastasis and sphere formation.

In conclusion, we identified miR-30a as a tumor suppressor miRNA in glioma, and low miR-30a expression may become an unfavorable prognostic factor in patients with glioma. Furthermore, miR-30a functions as the tumor suppressor in human glioma by targeting Wnt5a. These findings indicated that miR-30a acts as a potential target for treating glioma and the key function of miR-30a in glioma oncogenesis. It also has great value in early diagnosis, and in prognostic diagnosis. This study offers theoretical basis to better understand the molecular mechanism of glioma and its potential therapeutic strategies.

\section{Acknowledgements}

The present study was supported by the Natural Science Foundation of Gansu Province (no. 1606RJZA348) and the Science and Technology Project of Lanzhou city (2015-2-67).

\section{References}

1. Godlewski J, Newton HB, Chiocca EA and Lawler SE: MicroRNAs and glioblastoma; the stem cell connection. Cell Death Differ 17: 221-228, 2010.

2. Li Z, Wang H, Eyler CE, Hjelmeland AB and Rich JN: Turning cancer stem cells inside out: An exploration of glioma stem cell signaling pathways. J Biol Chem 284: 16705-16709, 2009.

3. Wen PY and Kesari S: Malignant gliomas in adults. N Engl J Med 359: 492-507, 2008.

4. Gladson CL, Prayson RA and Liu WM: The pathobiology of glioma tumors. Annu Rev Pathol 5: 33-50, 2010.

5. Kartha RV and Subramanian S: Competing endogenous RNAs (ceRNAs): New entrants to the intricacies of gene regulation. Front Genet 5: 8, 2014. eCollection.

6. Li M, Li J, Liu L, Li W, Yang Y and Yuan J: MicroRNA in human glioma. Cancers (Basel) 5: 1306-1331, 2013.

7. Zhang Y, Dutta A and Abounader R: The role of microRNAs in glioma initiation and progression. Front Biosci (Landmark Ed) 17: 700-712, 2012.

8. Wong JW: MicroRNA-induced silencing of glioma progression. J Neurosci 30: 3868-3869, 2010.

9. Wang Z, Wang B, Shi Y, Xu C, Xiao HL, Ma LN, Xu SL, Yang L, Wang QL, Dang WQ, et al: Oncogenic miR-20a and miR-106a enhance the invasiveness of human glioma stem cells by directly targeting TIMP-2. Oncogene 34: 407-419, 2015.

10. Zhao D, Jiang X, Yao C, Zhang L, Liu H, Xia H and Wang Y: Heat shock protein 47 regulated by miR-29a to enhance glioma tumor growth and invasion. J Neurooncol 118: 39-47, 2014.

11. Wan X, Cheng Q, Peng R, Ma Z, Chen Z, Cao Y and Jiang B ROCK1, a novel target of miR-145, promotes glioma cell invasion. Mol Med Rep 9: 1877-1882, 2014.

12. Guo M, Jiang Z, Zhang X, Lu D, Ha AD, Sun J, Du W, Wu Z, $\mathrm{Hu} \mathrm{L}$, Khadarian K, et al: miR-656 inhibits glioma tumorigenesis through repression of BMPR1A. Carcinogenesis 35: 1698-1706, 2014.

13. Zhang D, Yang G, Chen X, Li C, Wang L, Liu Y, Han D, Liu H, Hou X, Zhang W, et al: mir-300 promotes self-renewal and inhibits the differentiation of glioma stem-like cells. J Mol Neurosci 53: 637-644, 2014.

14. Yang TQ, Lu XJ, Wu TF, Ding DD, Zhao ZH, Chen GL, Xie XS, Li B, Wei YX, Guo LC, et al: MicroRNA-16 inhibits glioma cell growth and invasion through suppression of BCL2 and the nuclear factor- $\kappa \mathrm{B} 1 / \mathrm{MMP} 9$ signaling pathway. Cancer Sci 105: 265-271, 2014
15. Li SZ, Hu YY, Zhao J, Zhao YB, Sun JD, Yang YF, Ji CC, Liu ZB Cao WD, Qu Y, et al: MicroRNA-34a induces apoptosis in the human glioma cell line, A172, through enhanced ROS production and NOX2 expression. Biochem Biophys Res Commun 444: 6-12, 2014.

16. Zhang Y, Chen X, Lian H, Liu J, Zhou B, Han S, Peng B, Yin J, Liu W and He X: MicroRNA-503 acts as a tumor suppressor in glioblastoma for multiple antitumor effects by targeting IGF-1R. Oncol Rep 31: 1445-1452, 2014.

17. Chen Z, Li D, Cheng Q, Ma Z, Jiang B, Peng R, Chen R, Cao Y and Wan X: MicroRNA-203 inhibits the proliferation and invasion of U251 glioblastoma cells by directly targeting PLD2. Mol Med Rep 9: 503-508, 2014.

18. Alrfaei BM, Vemuganti R and Kuo JS: microRNA-100 targets SMRT/NCOR2, reduces proliferation, and improves survival in glioblastoma animal models. PLoS One 8: e80865, 2013.

19. Guo P, Nie Q, Lan J, Ge J, Qiu Y and Mao Q: C-Myc negatively controls the tumor suppressor PTEN by upregulating miR-26a in glioblastoma multiforme cells. Biochem Biophys Res Commun 441: 186-190, 2013.

20. Jiang J, Yang J, Wang Z, Wu G and Liu F: TFAM is directly regulated by miR-23b in glioma. Oncol Rep 30: 2105-2110, 2013.

21. Tu Y, Gao X, Li G, Fu H, Cui D, Liu H, Jin W and Zhang Y: MicroRNA-218 inhibits glioma invasion, migration, proliferation, and cancer stem-like cell self-renewal by targeting the polycomb group gene Bmil. Cancer Res 73: 6046-6055, 2013.

22. Zhang N, Wang X, Huo Q, Sun M, Cai C, Liu Z, Hu G and Yang Q: MicroRNA-30a suppresses breast tumor growth and metastasis by targeting metadherin. Oncogene 33: 3119-3128, 2014.

23. Kumarswamy R,Mudduluru G,Ceppi P,Muppala S, Kozlowski M, Niklinski J, Papotti M and Allgayer H: MicroRNA-30a inhibits epithelial-to-mesenchymal transition by targeting Snail and is downregulated in non-small cell lung cancer. Int J Cancer 130: 2044-2053, 2012

24. Zhu H, Wu H, Liu X, Li B, Chen Y, Ren X, Liu CG and Yang JM: Regulation of autophagy by a beclin 1-targeted microRNA, miR-30a, in cancer cells. Autophagy 5: 816-823, 2009.

25. Zhong M, Bian Z and Wu Z: miR-30a suppresses cell migration and invasion through downregulation of PIK3CD in colorectal carcinoma. Cell Physiol Biochem 31: 209-218, 2013.

26. Ouzounova M, Vuong T, Ancey PB, Ferrand M, Durand G, Le-Calvez Kelm F, Croce C, Matar C, Herceg Z and HernandezVargas H: MicroRNA miR-30 family regulates non-attachment growth of breast cancer cells. BMC Genomics 14: 139, 2013.

27. Zhao S, Ye X, Xiao L, Lian X, Feng Y, Li F and Li L: MiR-26a inhibits prostate cancer progression by repression of Wnt5a. Tumour Biol 35: 9725-9733, 2014.

28. Wu Y, Dong L, Bao S, Wang M, Yun Y and Zhu R: FK228 augmented temozolomide sensitivity in human glioma cells by blocking PI3K/AKT/mTOR signal pathways. Biomed Pharmacother 84: 462-469, 2016.

29. Shivdasani RA: MicroRNAs: Regulators of gene expression and cell differentiation. Blood 108: 3646-3653, 2006.

30. Cohen S: MicroRNAs in animal development. Editorial. Semin Cell Dev Biol 21: 727, 2010.

31. Zou Z, Ni M, Zhang J, Chen Y, Ma H, Qian S, Tang L, Tang J, Yao H, Zhao C, et al: MiR-30a can inhibit DNA replication by targeting RPA1 thus slowing cancer cell proliferation. Biochem J 473: 2131-2139, 2016.

32. Kumarswamy R1, Mudduluru G, Ceppi P, Muppala S, Kozlowski M, Niklinski J, Papotti $M$ and Allgayer $\mathrm{H}$ : MicroRNA-30a inhibits epithelial-to-mesenchymal transition by targeting Snail and is downregulated in non-small cell lung cancer. Int J Cancer 130: 2044-2053, 2012.

33. Cheng CW1, Wang HW, Chang CW, Chu HW, Chen CY, Yu JC, Chao JI, Liu HF, Ding SL and Shen CY: MicroRNA-30a inhibits cell migration and invasion by downregulating vimentin expression and is a potential prognostic marker in breast cancer. Breast Cancer Res Treat 134: 1081-1093, 2012.

34. Kikuchi A, Yamamoto H, Sato A and Matsumoto S: Wnt5a: Its signalling, functions and implication in diseases. Acta Physiol (Oxf) 204: 17-33, 2012.

35. Nishita M, Enomoto M, Yamagata K and Minami Y: Cell/tissuetropic functions of Wnt5a signaling in normal and cancer cells. Trends Cell Biol 20: 346-354, 2010.

36. McDonald SL and Silver A: The opposing roles of Wnt-5a in cancer. Br J Cancer 101: 209-214, 2009.

37. Katoh M: WNT signaling in stem cell biology and regenerative medicine. Curr Drug Targets 9: 565-570, 2008. 